\title{
MINIMAL ARCS IN METRIC SPACES
}

\section{S. K. HILDEBRAND and HAROLD WILLIS MILNES}

(Received 25 February 1972; revised 19 September 1972)

Communicated by E. Strzelecki

In this paper we discuss the existence of an arc of minimal length joining two arbitrary, yet fixed points in a complete metric space, where the metric is restricted only by the properties (A) and (B) given below. It is shown that under these conditions an arc of least length joining any two fixed points exists, and is unique. In addition, its length is shown to be equal to the metric distance between the points.

The additional restricting properties on the metric: $\rho$, are the following:

Property (A). Given any pair of points, $p, q$ with $\rho(p, q)=a$, for any $b \geqq 0, c \geqq 0, b+c=a$, and any $e>0$, there is a point $r$ such that $\rho(p, r) \leqq b+e$ and $\rho(r, q) \leqq c+e$.

We use the notations: $N_{p}(\alpha) \equiv\{x \mid \rho(p, x)<\alpha\}$ and $\operatorname{diam}[S]=\operatorname{lub}_{x, y \in S} \rho(x, y)$ here and subsequently throughout the paper.

Property (B). Given any pair of points $p, q$ and $\alpha>0$ such that $q \notin N_{p}(\alpha)$, for any $e>0$ there is a $\beta>0$ such that (i) $N_{p}(\alpha) \cap N_{q}(\beta) \neq \varnothing$, (ii) $\operatorname{diam}\left[N_{p}(\alpha)\right.$ $\left.\cap N_{k}(\beta)\right]<e$.

We remark that the octant surface of a sphere with the Euclidean metric in 3-space has property (B) but not property (A). A surface of a sphere with the same metric also fails to have property (B), as may be seen by considering $p, q$ to be any pair of opposite poles. As an example of a space with property (A) and not (B), we may consider the Cartesian plane with $\rho((x, y),(p, q))=$ $\max \{|x-p|,|y-q|\}$.

In the following theorems, for notational convenience, we refer the unit interval $I \equiv[J, 1]$ to a binary representation. We shall also, on occasion, mix ordinary fractions with this representation; for example $0.11+\frac{3}{8}$ which is $\frac{9}{8}$, or 1.001 in binary notation.

Theorem 1 illustrates that a complete metric space with a metric function having property (A) is arcwise connected. 
THEOREM 1. If $(R, \rho)$ is a complete metric space, and the metric $\rho$ has properties $(A)$, then $(R, \rho)$ is arcwise connected.

Proof. Let $\varepsilon>0$ be given, and $p_{0}, p_{1}$ be any two points of $R$, with $\rho\left(p_{0}, p_{1}\right)=d$. Set up the correspondence $p_{0}=f(0), p_{1}=f(1)$. With $a=d$, $b=c, p=p_{0}, q=p_{1}, e=\varepsilon / 2^{2}$, property (A) permits the selection of a point $p_{0.1}$ such that

$$
\rho\left(p_{0}, p_{0.1}\right)<\frac{d}{2}+\frac{\varepsilon}{2^{2}}<\frac{d}{2}+\frac{\varepsilon}{2} \text {, and } \rho\left(p_{0.1}, p_{1}\right)<\frac{d}{2}+\frac{\varepsilon}{2} \text {. }
$$

Set up the correspondence $p_{0.1}=f(0.1)$. Then, with $a=d / 2+\varepsilon / 2^{2}, b=c$, $e=\varepsilon / 2^{4}$, again applying property (A) find $p_{0.01}, p_{0.11}$ such that $\rho\left(p_{0.00}, p_{0.01}\right)$, $\rho\left(p_{0.01}, p_{0.10}\right), \rho\left(p_{0.10}, p_{0.11}\right)$,

$$
\rho\left(p_{0.11}, p_{1.00}\right)<\frac{d}{4}+\frac{\varepsilon}{2^{3}}+\frac{\varepsilon}{2^{4}}<\frac{d}{4}+\frac{\varepsilon}{2^{2}},
$$

and set up the correspondence $p_{0.01}=f(0.01), p_{0.11}=f(0.11)$. Proceeding inductively, $(n=1,2,3, \cdots)$ suppose that, with $\alpha_{s} \beta$ equal to 0 or $1, p_{\alpha_{0}, \alpha_{1} \ldots z_{n}}$, $p_{\beta_{0} \cdot \beta_{1} \cdots \beta_{n}}$ have been found, where $\beta_{0} \beta_{1} \beta_{2} \cdots \beta_{n}-\alpha_{0} \alpha_{1} \alpha_{2} \cdots a_{n}=2^{-n}$. Then, with

$$
a=\frac{d}{2^{n}}+\frac{\varepsilon}{2^{n+1}}+\frac{\varepsilon}{2^{n+2}}+\cdots+\frac{\varepsilon}{2^{2 n}}, b=c, e=\varepsilon / 2^{2 n+2},
$$

property (A) permits the selection of $p_{\alpha_{0} \cdot \alpha_{1} \cdots \alpha_{n}}$, such that

$\rho\left(p_{\alpha_{0}, \alpha_{1} \cdots \alpha_{n}}, p_{\alpha_{0} \cdot \alpha_{1} \cdots \alpha_{n} 1}\right)<\frac{d}{2^{n+1}}+\frac{\varepsilon}{2^{n+1}}+\cdots+\frac{\varepsilon}{2^{2 n+1}}+\frac{\varepsilon}{2^{2 n+2}}<\frac{d}{2^{n+1}}+\frac{\varepsilon}{2^{n}}$.

The correspondence $p_{\alpha_{0}, \alpha_{0} \cdots \alpha_{n} 1}=f\left(\alpha_{0}, \alpha_{1} \ldots \alpha_{n} 1\right)$ is made.

We show that if $p_{a_{0} . a_{1} \cdots a_{k}}, p_{b_{0} . b_{1} \cdots b_{1}},(a, b=0$ or $1 ; k, l<\infty)$ are two points with $b_{0} \cdot b_{1} \cdots b_{l} \geqq a_{0} . a_{1} \cdots a_{k}$ then

$$
\rho\left(p_{b_{0}, b_{1} \cdots b_{l}}, p_{a_{0} \cdot a_{1} \cdots a_{k}}\right)<\left(b_{0} . b_{1} \cdots b_{l}-a_{0} . a_{1} \cdots a_{k}\right)(d+\varepsilon) .
$$

Let $\sigma=|l-k|$ and consider the sequence of numbers

$$
\left\{a_{0} \cdot a_{1} \cdots a_{k}+j / 2^{\sigma} \mid j=0,1,2, \cdots, s ; s=\left[b_{0} \cdot b_{1} \cdots b_{l}-a_{0} \cdot a_{1} \cdots a_{k}\right] 2^{\sigma}\right\}
$$

with their associated images $p_{a_{0} . a_{1} \cdots a_{k}+j / 2 \sigma}$. Then:

$$
\begin{gathered}
\rho\left(p_{b_{0}, b_{1} \cdots b_{l}}, p_{a_{0} \cdot a_{1} \cdots a_{k}}\right) \leqq \sum_{j=0}^{s-1} \rho\left(p_{a_{0} \cdot a_{1} \cdots a_{k}+j / 2^{\sigma}},\right. \\
\left.p_{a_{0} \cdot a_{1} \cdots a_{k}+(j+1) / 2 \sigma}\right) \leqq \sum_{j=0}^{s-1}\left(\frac{d}{2^{\sigma}}+\frac{\varepsilon}{2^{\sigma}+1}+\cdots+\frac{\varepsilon}{2^{2 \sigma}}\right) \\
<\sum_{j=0}^{s-1}\left(\frac{d}{2^{\sigma}}+\frac{\varepsilon}{2^{\sigma}}\right)=\frac{s}{2^{\sigma}}(d+\varepsilon)=\left(b_{0} \cdot b_{1} \cdots b_{l}-a_{0} \cdot a_{1} \cdots a_{k}\right)(d+\varepsilon) .
\end{gathered}
$$

The function $\rho$ satisfies $\rho(p(x), \rho(y))<|x-y|(d+\varepsilon)$ for all $x$ and $y$ in a dense 
subset $K$ of $I$. Thus $\rho$ is uniformly continuous on $K$ and has a unique continuous extension to $I$.

We adopt the following definition of arc length, that will be used in subsequent theorems.

Definition. If $S \equiv f[I]$ is an arc, its length is defined as

$$
l(S)=\sup _{\left\{x_{i}\right\}} \sum_{i=0}^{N-1} \rho\left(f\left(x_{i}\right), f\left(x_{i+1}\right)\right)
$$

where $\left\{x_{i} \mid 0=x_{0}<x_{1}<\cdots<x_{N}=1\right\}$ is any partition of I. If $l(S)<\infty$, then $S$ has finite length; otherwise $l(S)$ is infinite.

THEOREM 2. If $(R, \rho)$, a complete metric space, has properties (A) and (B), and $p, q \in R$ with $\rho(p, q)=d$, then there is an arc $f(I)$ joining $p$ and $q$ such that $\rho(f(x), f(y))=d|x-y|$ for all $x, y \in I$.

Proof. Let $p_{0}, p_{1}$ be the two points of $R$. We shall use the same notations as in the proof of theorem 1. Let $f(0)=p_{0}, f(1)=p_{1}$. The procedure for establishing the correspondence $f: I \rightarrow S$ is similar to that of theorem 1, with some modification. Suppose that $p_{\alpha_{0}, \alpha_{1} \cdots \alpha_{n}}, p_{\beta_{0} . \beta_{1} \cdots \beta_{n}}$ have been found, and that $\beta_{0} \cdot \beta_{1} \cdots \beta_{n}-\alpha_{0} \cdot \alpha_{1} \cdots \alpha_{n}=2^{-n}$. Using properties (A) and (B), putting $a=d / 2^{n}$, $b=c$, letting $\varepsilon_{\gamma}=2^{-\gamma},(\gamma=1,2, \cdots)$, and choosing $\varepsilon^{\prime}>0$ sufficiently small, pick

$$
\begin{aligned}
& p_{\gamma} \in N p_{\alpha_{0}, \alpha_{1} \cdots \alpha_{n}}\left(b+\varepsilon^{\prime}\right) \cap N p_{\beta_{0} . \beta_{1} \cdots \beta_{n}}\left(c+\varepsilon^{\prime}\right) \text { with: } \\
& \quad \operatorname{diam}\left[N p_{\alpha_{0} . \alpha_{1} \cdots \alpha_{n}}\left(b+\varepsilon^{\prime}\right) \cap N p_{\beta_{0} \cdot \beta_{1} \cdots \beta_{n}}\left(c+\varepsilon^{\prime}\right)\right]<\varepsilon_{\gamma}
\end{aligned}
$$

If $\mu, \lambda \geqq \gamma$, then $\rho\left(p_{\mu}, p_{\lambda}\right)<\varepsilon_{y}$. By completeness the sequence $\left\{p_{\gamma}\right\}$ has a limit which we denote as: $p_{\alpha_{0}, \alpha_{1} \cdots \alpha_{n} 1}$. We make the correspondence $p_{\alpha_{0}, \alpha_{1} \cdots \alpha_{n} 1}=$ $f\left(\alpha_{0} \cdot \alpha_{1} \cdots \alpha_{n 1}\right)$.

In a metric space $\rho\left(q_{y}, r\right) \rightarrow \rho\left(q_{0}, r\right)$ when $q_{\gamma} \rightarrow q_{0},(\gamma=1,2, \cdots)$. With $q_{\gamma}=p_{\gamma}, q_{0}=p_{\alpha_{0}, \alpha_{1} \cdots \alpha_{n} 1}$ and $r$ alternately equal to $p_{\alpha_{0}, \alpha_{1} \cdots \alpha_{n}}$ and $p_{\beta_{0}, \beta_{1} \cdots \beta_{n}}$, it follows that $\rho\left(p_{\alpha_{0}, \alpha_{1} \cdots a n 1}, r\right)=b=c=a / 2=d / 2^{n+1}$.

The construction of $S$ and the proof of the continuity of $f$ follow the technique of theorem 1.

We consider two points $p_{a_{0} \cdot a_{1} \cdots a_{k}}$ and $p_{b_{0} \ldots b_{1}}$ which correspond to terminating binary decimals:

$$
b_{0} \cdot b_{1} \cdots b_{l} \geqq a_{0} \cdot a_{1} \cdots a_{k} .
$$

We show $\rho\left(p_{b_{0}, b_{1} \cdots b_{l}}, p_{a_{0} . a_{1} \cdots a_{k}}\right)=\left(b_{0} \cdot b_{1} \cdots \quad b_{l}-a_{0} \cdot a_{1} \cdots a_{k}\right) d$. Let $\sigma=|l-k|$ and consider the sequence of numbers:

$$
\left\{a_{0} \cdot a_{1} \cdots a_{k}+j / 2^{\sigma} \mid j=0,1,2, \cdots, s ; s=\left(b_{0} \cdot b_{1} \cdots b_{l}-a_{0} \cdot a_{1} \cdots a_{k}\right) 2^{\sigma}\right\}
$$

with their associated images $p_{a_{0}, a_{1} \cdots a_{k}+j / 2^{\sigma}}$. Then, as above:

$$
\rho\left(p_{a_{0}, a_{1} \cdots a_{k}+j / 2 \sigma}, p_{a_{0} a_{1} \cdots a_{k}+(j+1) / 2 \sigma}\right)=d / 2^{\sigma}
$$


Therefore:

$$
\rho\left(p_{a_{0}, a_{1} \cdots a_{k}}, p_{b_{0} . b_{1} \cdots b_{l}}\right) \leqq s d / 2^{\sigma}=\left(b_{0} \cdot b_{1} \cdots b_{l}-a_{0} \cdot a_{1} \cdots a_{k}\right) d
$$

By a similar argument, it follows that:

But:

$$
\begin{aligned}
\rho\left(p_{0}, p_{a_{0} . a_{1} \cdots a_{k}}\right) & \leqq\left(a_{0} . a_{1} \cdots a_{k}\right) d \\
\rho\left(p_{b_{0} . b_{1} \cdots b_{1}}, p_{1}\right) & \leqq\left(1-b_{0} . b_{1} \cdots b_{\imath}\right) d
\end{aligned}
$$

$$
\begin{aligned}
d=\rho\left(p_{0}, p_{1}\right) & \leqq \rho\left(p_{0}, p_{a_{0} \cdot a_{1} \cdots a_{k}}\right)+\rho\left(p_{a_{0} \cdot a_{1} \cdots a_{k}}, p_{b_{v} \cdot b_{1} \cdots b_{l}}\right)+\rho\left(p_{b_{0} \cdot b_{1} \cdots b_{l}}, p_{1}\right) \\
& \leqq d\left[a_{0} \cdot a_{1} \cdots a_{k}+\left(b_{0} \cdot b_{1} \cdots b_{l}-a_{0} . a_{1} \cdots a_{k}\right)+\left(1-b_{0} \cdot b_{1} \cdots b_{l}\right)\right]=d
\end{aligned}
$$

so that:

$$
\begin{aligned}
& \rho\left(p_{a_{0} \cdot a_{n} \cdots a_{k}}, p_{b_{0} \cdot b_{1} \cdots b_{l}}\right)= \\
& \quad=d-\rho\left(p_{0}, p_{a_{0}, a_{1} \cdots a_{k}}\right)-\rho\left(p_{b_{0}, b_{1} \cdots b_{l}}, p_{1}\right) \geqq d\left[1-a_{0} \cdot a_{1} \cdots a_{k}-\left(1-b_{0} \cdot b_{1} \cdots b_{l}\right)\right] .
\end{aligned}
$$

Combining this with equation (1) and noting that if $\left\{q_{\gamma}\right\},\left\{r_{\gamma}\right\},(\gamma=1,2, \cdots)$ are any two sequences of points with terminating representations that tend to $q_{a}, q_{b}$ in the limit, then $\rho\left(q_{a}, q_{b}\right)=d|b-a|$, the stated conclusion follows.

Corollary (i). The arc $f(I)$ in theorem 2 has minimum length and $l(f(I))=\rho(p, q)$.

Proof. Suppose that $\left\{x_{i} \mid i=0,1, \cdots, N\right\}$ is any partition of $I$; then

$$
\sum_{i=0}^{N-1} \rho\left(f\left(x_{i}\right), f\left(x_{i+1}\right)\right)=d \sum_{i=0}^{N-1}\left(x_{i+1}-x_{i}\right)=d\left(x_{N}-x_{0}\right)=d .
$$

Therefore $l(S)=d$. Since the length of any arc joining $p_{0}$ to $p_{1}$ is at least $\rho\left(p_{0}, p_{1}\right)$ we have proven the corollary.

COROLlary (ii) Let $r$ be any third point on the arc $f(I)$ joining $p$ and $q$, and let $S_{0}$ and $S_{1}$ be the subarcs of $f(I)$ from $p$ to $r$, and $r$ to $q$. Then $S_{0}$ and $S_{1}$ have minimum length and $l\left(S_{0}\right)=\rho(p, r)$ and $l\left(S_{1}\right)=\rho(r, b)$.

Proof. By Theorem 2 and Corollary (i) there is an arc $T_{0}$ of length $\rho(p, r)$ joining $p$ to $r$; and there is an $\operatorname{arc} T_{1}$, joining $r$ to $f$ of length $\rho(r, f)$. Also $l\left(T_{0}\right) \leqq l\left(S_{0}\right), l\left(T_{1}\right) \leqq l\left(S_{1}\right)$. Therefore:

$$
\rho(p, r)+\rho(r, q)=l\left(T_{0}\right)+l\left(T_{1}\right) \leqq l\left(S_{0}\right)+l\left(S_{1}\right)=l(f(I))=\rho(p, q) .
$$

But the triangle inequality implies

$$
\rho(p, r)+\rho(r, q) \geqq \rho(p, q) .
$$

Therefore: $l\left(T_{0}\right)+l\left(T_{1}\right)=l\left(S_{0}\right)+l\left(S_{1}\right)$. Suppose $l\left(T_{0}\right)<l\left(S_{0}\right)$ then $l\left(T_{1}\right)>l\left(S_{1}\right)$ and there is a contradiction. Similarly if $l\left(T_{1}\right)<l\left(S_{1}\right)$.

THEOREM 3. If $(R, \rho)$, a complete metric space, has properties (A) and (B) and if $p_{0}, p_{1}$ are any two points, then any arc of least length joining $p_{0}$ to $p_{1}$ 
intersects the locus of points $C_{\alpha} \equiv\left\{x \mid \rho\left(p_{0}, x\right)=\alpha, 0 \leqq \alpha \leqq \rho\left(p_{0}, p_{1}\right)\right\}$ in exactly one point.

Proof. Since any arc of least length joining $p_{0}$ to $p_{1}$ is a continuum, it must have at least one point in common with $C_{\alpha},\left(0 \leqq \alpha \leqq \rho\left(p_{0}, p_{1}\right)\right)$. Suppose there are two points $q_{1}, q_{2}$ common to $S$ and $C_{\alpha}$. Without loss of generality, let $q_{1}$ be the first point according to the mapping of the arc from the unit interval to $S_{0}$. If $S_{0}$ denotes the subarc of $S$ from $p_{0}$ to $q_{1}$, then corollary (ii) of theorem 2 shows that $l\left(S_{0}\right)=\rho\left(p_{0}, p_{1}\right)=\alpha$. If $S_{1}$ denotes the subarc of $S$ from $q_{2}$ to $p_{1}$, then the same corollary shows that $l\left(S_{1}\right)=\rho\left(p_{0}, p_{1}\right)-\alpha$. Hence, the subarc of $S$ from $q_{1}$ to $q_{2}$ must have zero length; or $q_{1}=q_{2}$.

THEOREM 4. If $(R, \rho)$, a complete metric space, has properties (A) and (B) then the minimizing arc joining any two points $p_{0}, p_{1}$ is unique up to parametric representation.

Proof. Let $S$ be the arc of least length according to the construction of Theorem 2. Assume that $T$ is any other arc with $l(T)=l(S)$. Let $C_{\alpha} \equiv\left\{x \mid \rho\left(p_{0}, x\right)=\alpha\right\}$. By theorem 3, $C_{\alpha} \cap S$ and $C_{\alpha} \cap T$ are singleton sets, for $0 \leqq \alpha \leqq \rho\left(p_{0}, p_{1}\right)$. If $C_{\alpha} \cap S \equiv C_{\alpha} \cap T$ for all $0 \leqq \alpha \leqq \rho\left(p_{0}, p_{1}\right)$, then $T$ is merely a different parametric representation of $S$. Suppose therefore that for some $\alpha, C_{\alpha} \cap S=q \neq r=C_{\alpha} \cap T$. Then $\rho(p, r)=k \neq 0$. Using the notation introduced in the definition of property (B), by property (B) there exists an $\varepsilon>0$ such that:

$$
\operatorname{diam}\left[N_{p 0}(\alpha) \cap N_{p_{1}}(d-\alpha+\varepsilon)\right]<k / 2 \text {. }
$$

We note that $\operatorname{diam}\left[\overline{N_{p_{0}}(\alpha) \cap N_{p_{1}}(d-\alpha+\varepsilon)}\right]<k / 2$. Now $q \in N_{p_{1}}(d-\alpha+\varepsilon)$ and since $q \in C_{\alpha}$, therefore $q \in \overline{N_{p_{0}}(\alpha)}$. By an elementary theorem of topology, if $A, B$ are subsets of a topological space and $A$ is open, then $A \cap \bar{B} \subseteq \overline{A \cap B}$ noting that $N_{p_{1}}(d-\alpha+\varepsilon)$ is open, it follows that:

$$
q \in \overline{N_{p_{0}}(\alpha) \cap N_{p_{1}}(d-\alpha+\varepsilon)}
$$

But $r \notin \overline{N_{p_{0}}(\alpha) \cap N_{p_{1}}(d-\alpha+\varepsilon)}$, since $\rho(q, r)>k / 2$ and $\operatorname{diam} \overline{\left[N_{p_{0}}(\alpha) \cap N_{p_{1}}(d-\alpha+\varepsilon)\right]}$ $<k / 2$. However, $r \in \overline{N_{p_{0}}(\alpha)}$, so that by the above cited theorem, $r \notin N_{p_{1}}(d-\alpha+\varepsilon)$ Therefore $\rho\left(p_{1}, r\right) \geqq d-\alpha+\varepsilon$.

If $T_{0}$ is the subarc of $T$ from $p_{0}$ to $r$, and $T_{1}$ is that from $r$ to $p_{1}$, then:

$$
d=l\left(T_{0}\right)+l\left(T_{1}\right) \geqq \rho\left(p_{0}, r\right)+\rho\left(r, p_{1}\right)=\alpha+\rho\left(p_{1}, r\right) \geqq \alpha+(d-\alpha+\varepsilon)=d+\varepsilon .
$$

This is a contradiction, and hence $S$ is the unique arc of minimal length joining $p_{0}$ to $p_{1}$.

\section{Texas Tech University}

Lubbock, Texas 79409, U.S.A. 